\title{
EDITORIAL
}

\section{NEW INSIGHT FOR MASKLESS LITHOGRAPHY}

Two significant microlithography meetings took place in 2005: The ML2 workshop organized by International Sematech and the 2005 SPIE Microlithography Symposium. In the former meeting, more than 20 articles were presented or posted in one and half days. In the latter meeting, 6 articles on maskless lithography were presented or posted out of a total of 738 for the entire symposium. The total time it took was I hour and 20 minutes. Is ML2 neglected in the microlithography community?

A common outcry from the maskless researchers is that there is no market for the maskless technology. If the goal is to address mask cost, maskless lithography is indeed limited in market potential. In fact, the conventional mask-making industry is already facing the problem of a small market, making its development costly. Unless ML2 completely replaces the use of masks, the situation will become worse. The cost of ML2 adds to the financial burden of the semiconductor industry while the mask-making market reduced by ML2 further drives up the cost of developing masking tools and technology.

There are many applications for ML2, other than the common wisdom of replacing the masks that print only a small number of wafers. Each application needs a certain type of maskless technology. ML2 can aim at becoming the next generation lithography (NGL). It can also aim at a dual application tool that can make masks and can write directly on wafer; ditto for a tool that provides next generation imaging before the mask-making and wafer imaging technologies are developed.

In the long run, the most desirable ML2 would be a NGL to succeed optical lithography. Given that any form of NGL replication technology, either e-beam or EUV, cannot support the mask pellicle, ML2 eliminates that problem completely. Replacing the replication technology also removes the need for making a mask. But, how about throughput and cost? One has to be realistic that it will be difficult to develop a single tool that can rival the throughput of a single optical scanner, namely 100-150 wafers per hour. The correct economic criteria for ML2 should be similar throughput, cost, and footprint of the NGL tool being replaced, regardless of the number of tools or beams that ML2 uses. One should not underestimate the shrinking power of MEMS techniques and the data processing power by the time of the node that requires a ML2 NGL. Resorting to e-beam ML2, the gigantic vacuum-tube-style e-beam column will go the way of vacuum tubes in the electronics industry. MEMS techniques can produce thousands of beams and their controllers at high reproducibility and relatively low cost. Cleverly optimizing the number and location of the beams and tools can reduce the data rate required. Massive parallelism in the beams and the data processing units will escalate until the cost target is reached. When performance and economy meet the requirements, the market of this ML2 application will be huge.

The second type of ML2 does not need the same wafer throughput as a NGL tool. Five to ten wafers per hour suffice. It should facilitate prototyping for new tape outs to shorten the design iteration cycle, saving the mask-making time as well as mask-making cost for small prototype lots. To avoid the problem of the mask-making cost climbing further because of less demand due to this ML2 tool, it should also have mask-making capability. This way the two capabilities help each other to support the smaller market demand in each category as compared to a full-fledged NGL tool. There is an advantage of making it a dual-purpose tool, besides just flexibility. Most of the system development, debugging, as well as process optimization and system maintenance in the factory or in the field can be performed with wafers to save the cost of using mask substrates. Prototyping requires that the tool can expose the resist that will be used for mass production using a replicating tool. The resist image contrast and profile should mimic that from the replicating tool. The market for this tool is much larger than that for mask-making tools in a given generation. One should note that this ML2 application saves the development cost of the mask writer but not inspection and repair. The latter can arguably be avoided by using previous-generation repair tools and take advantage of the much higher throughput of the ML2 tool to produce a mask without unrepairable defects out of many writings.

The third type of ML2 can make use of an even lower throughput. One to three wafers per hour serves the purpose. The resolution of this tool should be adjustable for a few nodes beyond the present ones. Trading resolution with throughput is acceptable. For the same considerations as the second type, this tool should also be able to expose masks at a few nodes. The capability to expose resists for mass production is preferred. However, this cannot be held too strictly, because only a general resist category can be defined due to the absence of the to-be-developed mass-replicating tool. Because of the cross-generation capability, the market for this type of tool is not negligible.

There are indeed markets for ML2.

\section{$\mathrm{JM}^{3}$ Letters and Communications}

I would like to bring to your attention the $\mathrm{JM}^{3}$ Letters section that was instated in July 2003. This section is intended for rapid publication of short technical communications of significant interest in the field of microlithography, microfabrication, and microsystems. A $\mathrm{JM}^{3}$ letter may be a piece of valuable work that the authors do not have time to expand into a full paper. Or, 
the authors might like the microcommunity to know about the valuable work before they spend more time to complete a full paper. Letters will be reviewed with the same rigor as journal articles but with an accelerated pace. Accepted letters will be expedited for publication in the next available issue. With the introduction of e-First publication at SPIE, the publication of these letters is even quicker than before. They will appear electronically first then in the printed journal. When you have something significant and want the micro-community to know quickly, please use this format. We consider these letters prestigious enough to warrant special attention to them. Links to letters published in $\mathrm{JM}^{3}$ will also be included in the soon-to-be-launched SPIE Letters virtual journal (an online compilation of letters published in the four SPIE journals), providing wider accessibility. The manuscript length of $\mathrm{JM}^{3}$ letters may not exceed three printed journal pages. A brief cover letter setting forth the significance of the paper must accompany the manuscript.

Another section for short articles is Communications. The purpose of this section is to publish short communications on original work, comments on recent papers, and errata. Contributions must be technical in nature. Original work will be reviewed with the same rigor as journal articles.

Happy reading!

\section{Burn J. Lin Editor-in-Chief}

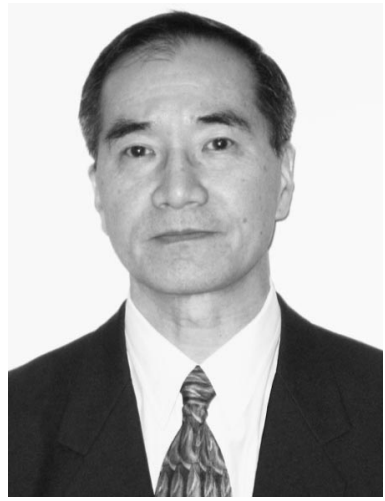

